\title{
Product formation in rhodopsin by fast hydrogen motions $\dagger$
}

\author{
Oliver Weingart, ${ }^{* a}$ Piero Altoè, ${ }^{b}$ Marco Stenta, ${ }^{c}$ Andrea Bottoni, ${ }^{b}$ Giorgio Orlandi ${ }^{b}$ and \\ Marco Garavelli $* b$
}

Received 11th November 2010, Accepted 22nd December 2010

DOI: $10.1039 /$ c0cp02496a

The photochemical cis-trans isomerization of retinal in rhodopsin is investigated by structure sampling and excited state QM/MM trajectories with surface hopping. The calculations uncover the motions responsible for photoproduct formation and elucidate the reasons behind the efficient photoisomerization in the primary event of visual transduction.

Visual perception is one of the most fascinating photochemical processes devised by nature. The rhodopsin protein (Rh), through photoinduced molecular deformations, converts with remarkable efficiency the energy of a single photon into chemical energy, eventually leading to a nerve impulse and vision. ${ }^{1,2}$ Its primary event involves an ultrafast (200 fs) and efficient (0.65 quantum yield) cis-trans isomerisation of the 11-cis retinal protonated Schiff base (PSB) chromophore. In solvent, the quantum yield is more than three times smaller ${ }^{3}$ and the photoisomerization is slower by a factor of 10 . Clarifying the mechanism behind this ultrafast and efficient reaction is crucial for a general understanding of efficient and fast biological molecular photo-switches, and for exploiting these properties in artificial/bio-mimetic systems. ${ }^{4}$

This photoreaction has been studied in the past both in vacuo $^{5-7}$ and in the protein binding pocket. ${ }^{8-12}$ All calculations support an ultrafast dynamics through a conical intersection (CI), which directly connects the excited and ground state potentials of the retinal chromophore.

Very recent hybrid QM(CASSCF)/MM simulations in $\mathrm{Rh}^{13}$ have produced experimentally accurate transient sub-20 fs spectroscopies of the CI dynamics and primary visual event (i.e., the $200 \mathrm{fs}$ photorhodopsin formation) that are supportive of a space saving photoisomerization mechanism reminiscent of Warshel's bicycle pedal model. ${ }^{14}$ These findings alone, however, can not explain why the photoisomerization in rhodopsin is so uniquely efficient. The remarkable success of this process must be connected with characteristic geometrical

${ }^{a}$ Max-Planck-Institut für Kohlenforschung, Kaiser-Wilhem-Platz 1, 45470 Mülheim an der Ruhr, Germany.

E-mail:Weingart@mpi-muelheim.mpg.de

${ }^{b}$ Dipartimento di Chimica "G. Ciamicia", Universita' degli Studi di

Bologna, Via Selmi 2, 40126 Bologna, Italy.

E-mail: marco.garavelli@unibo.it

${ }^{c}$ Ecole Polytechnique Fédérale de Lausanne, SV IBII UPDALPE,

AAB 017, Station 15, CH-1015, Lausanne, Switzerland

$\dagger$ Electronic supplementary information (ESI) available: Computational

details, discussion and graphs on statistical analysis, energy storage and

limitations of methods. See DOI: 10.1039/c0cp02496a

changes which assure that at the reactive point (the CI) the wave packet is directed mainly toward the photoproduct side of the potential energy surface, i.e. the all-trans retinal. Candidates for these motions are the internal coordinates which change the dihedrals of the isomerizing double bond, i.e. the $\mathrm{C} 11=\mathrm{C} 12$ carbon skeletal twisting, but also torsion of the $\mathrm{C} 11$ and $\mathrm{C} 12$ hydrogens, which is related to the hydrogen out-of-plane (HOOP) mode at this bond. Participation of the HOOP mode has been supposed first by Mathies et al., based on the analysis of Raman spectra. ${ }^{15} \mathrm{~A}$ direct connection between the quantum yield and the HOOP motion has been drawn in earlier studies of pre-twisted retinal models in vacuo, where an even higher quantum yield $(0.75)$ has been reported than in rhodopsin. ${ }^{16}$

By employing an extended sample of hybrid QM(CASSCF)/ $\mathrm{MM}$ trajectories at physiological conditions (50 trajectories, $300 \mathrm{~K}$ ), here we analyse the motions of the rhodopsin photoreaction leading to the product (11-trans) and educt (11-cis) sides of the potential energy surface, respectively. A strong involvement of the $\mathrm{H}-\mathrm{C} 11=\mathrm{C} 12-\mathrm{H}$ coordinate is observed. This motion (actually, its change in time) is correlated to the branching into cis-educt and transphotoproduct portions in the product determining step of the photoreaction. Eventually, for efficient product generation, it is important when the molecules hit the conical intersection seam and hop to the ground state: the earlier this event occurs, the higher is the quantum yield.

QM/MM trajectory calculations have been performed with the COBRAMM ${ }^{17}$ interface, combining the Molpro ${ }^{18}$ and Amber ${ }^{19}$ programs. For details on the protein setup and computations please refer to the Supporting Information (SI). Briefly, a state average three root CASSCF (10/10)/ 6-31G* approach has been used for the six double bonds of the polyene chain and the $\mathrm{CH}_{2}$-group of lysine (Fig. 1). The remaining retinal and protein atoms were treated with the Amber ff99 force field. Link-atoms were used to connect QM and $\mathrm{MM}$ regions. The chromophore and two neighbouring water molecules were free to move in the simulations, while all other atoms were fixed at their positions in the protein crystal (PDBfile 1U19). ${ }^{20}$

After ground state structure optimization and subsequent numerical frequency calculation, starting conditions were obtained from vibrational mode sampling at $300 \mathrm{~K}$, excluding the fast $\mathrm{C}-\mathrm{H}$ and $\mathrm{N}-\mathrm{H}$ stretching modes. For nuclear propagation the velocity Verlet integrator was used 


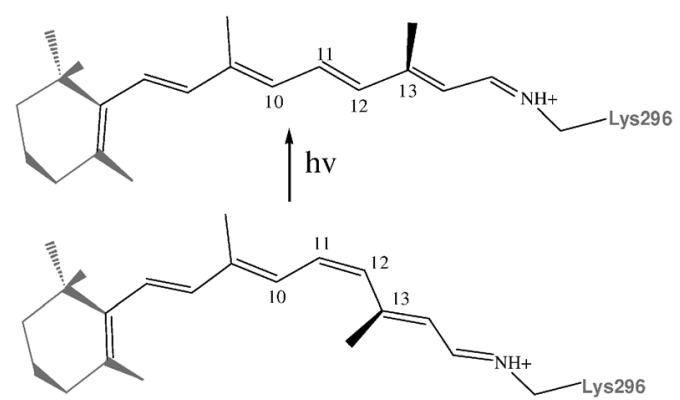

Fig. 1 Light-induced 11-cis to all-trans photo-conversion of retinal PSB in Rhodopsin. Black lines indicate the size of the QM region in the present molecular dynamics calculations; the grey coloured section and the remaining rhodopsin protein were treated at the Amber MM level.

with a time step of $0.5 \mathrm{fs}$, reduced to $0.25 \mathrm{fs}$ in the vicinity of an $\mathrm{S}_{1} / \mathrm{S}_{0}$ crossing. Molpro's CPMCSCF routines were employed in order to accurately calculate state averaged (SA) CASSCF gradients. Trajectories were brought to the ground state, when the scalar product of the $\mathrm{S}_{1}$ and $\mathrm{S}_{0}$ state coefficients indicated that a surface crossing had been passed..$^{21}$ The trajectories were then propagated on $\mathrm{S}_{0}$, until the final photoproduct could be identified from the evolution of the $\mathrm{C} 11=\mathrm{C} 12$ dihedral angles.

The minimum energy path along the torsion coordinate from the FC point to the CI (see SI) is barrierless, ${ }^{22}$ and the trajectories essentially follow this path. Following photoexcitation to $\mathrm{S}_{1}$, the first motion is along stretching coordinates. Thus, coherent $\mathrm{C}=\mathrm{C}$ stretching motions are impulsively generated out of the Franck-Condon region, that anticipate rotation of both, the $\mathrm{C} 11=\mathrm{C} 12$ and $\mathrm{C} 9=\mathrm{C} 10$ bonds. At 70 fs into the simulation the majority of molecules has already a largely twisted $\mathrm{C} 11=\mathrm{C} 12$ bond $\left(\mathrm{ca} .70^{\circ}\right.$ on average, see also Fig. 2). Also the $\mathrm{C} 9=\mathrm{C} 10$ torsion has evolved by this time, but somewhat slower, from $\mathrm{ca} .170^{\circ}$ to $210^{\circ} . \mathrm{C} 11=\mathrm{C} 12$ carbon skeletal torsion proceeds essentially parallel to $\mathrm{H}-\mathrm{C}=\mathrm{C}-\mathrm{H}$ motion at the same atoms. A branching into cis- and trans fractions becomes apparent at around $100 \mathrm{fs}$, when a major portion of trajectories hops to the electronic ground state (the average $S_{1}$ excited state lifetime is $c a$. $110 \mathrm{fs}$ ). While a minor fraction of trajectories (ca. 20\%) arrives directly at a CI and decays to the ground state within 50-80 fs, the majority of molecules approach the crossing region several times, before they finally hit the seam and hop to the lower state. The molecules hit the CI seam between $c a$. -65 and $-105^{\circ}$ of $\mathrm{C} 10-\mathrm{C} 11=\mathrm{C} 12-\mathrm{C} 13$ torsion, which is located $c a$. $17 \mathrm{kcal} \mathrm{mol}^{-1}$ below the energy of the $\mathrm{FC}$ point. On average it takes three to four $S_{1} / S_{0}$ approaches, until the molecules encounter the crossing seam and decay. After the first approach of the $S_{1} / S_{0}$ surfaces, we note rapid oscillations in the $\mathrm{H}-\mathrm{C}=\mathrm{C}-\mathrm{H}$ torsion angle in the majority of trajectories.

The $\mathrm{C} 9=\mathrm{C} 10$ bond stops rotating after the surface hop, and either reverts in the cis-branch or stays twisted in trans (Fig. 2). On average this bond twists by $\mathrm{ca} .50^{\circ}$ (from $170^{\circ}$ to $220^{\circ}$ ) until the decay point. The average value for the $\mathrm{C} 11=\mathrm{C} 12$ bond torsion is $c a .-90^{\circ}$ at hopping, consistent with constrained geometry optimizations of retinal in rhodopsin. ${ }^{22}$ In the trans branch the $\mathrm{C} 11=\mathrm{C} 12$ bond persists at values of $c a .-140^{\circ}$. Large oscillations of the bond dihedrals indicate activation of the $\mathrm{C} 11=\mathrm{C} 12$ and $\mathrm{C} 9=\mathrm{C} 10$ torsional modes in the cis fraction, while the out-of plane motions appear damped on the photoproduct side of the potential.

The $c a$. 0.6 calculated quantum yield in this set is in good qualitative agreement with the experimental result, considering that the surface hopping method we have applied accounts only for hops through the $S_{1} / S_{0}$ crossing seam.

Fig. 3 shows an overlay of the averaged hopping structures that lead to the cis and trans photoproducts. There is no obvious difference in the two geometries, i.e. the hopping structure is not the factor governing the fate of the molecule. A similar conclusion can be drawn from the distribution of the $\mathrm{C} 10-\mathrm{C} 11=\mathrm{C} 12-\mathrm{C} 13$ torsion angles and the corresponding $\mathrm{H}-\mathrm{C} 11=\mathrm{C} 12-\mathrm{H}$ torsions. Thus, the product determining step can not exclusively be attributed to the absolute value of a geometry parameter at the surface hop, and branching to cis or trans paths can occur from the same geometry.

The upper graph of Fig. 4 depicts the movement of the $\mathrm{C} 11=\mathrm{C} 12$ hydrogen and carbon atoms at the point of surface hopping by means of their dihedral angle gradient. Carbon skeletal torsion appears to play a minor role in photoproduction. This is visible in the lower right section, where nine trajectories lead to the cis photoproduct, even at strongly increasing $\mathrm{C} 10-\mathrm{C} 11=\mathrm{C} 12-\mathrm{C} 13$ torsion. Apparently, the hydrogen coordinate gives a better correlation. With only few exceptions the left side of the graph, where this angle is increasing, shows mainly trans photoproducts. In some cases the hydrogen motion is overruled by other modes, e.g. by carbon torsion in the upper left section of the graph. It should be noted that the HOOP coordinate, which is generally defined as the difference of hydrogen and carbon torsions, gives a comparable but slightly reduced correlation (see SI).

In short living trajectories that hop at the first close encounter of the $S_{1} / S_{0}$ surfaces we note an enhanced probability of yielding the trans photoproduct. This is also visible from the lower graph in Fig. 4, where the gradients of the two dihedral angles are plotted at the point where the $S_{1}$ and $\mathrm{S}_{0}$ surfaces approach for the first time. The lower left section of the graph again corresponds to increase in both dihedrals. Notably, the number of trajectories in this section is considerably higher than in the upper graph. Accordingly, this would suggest a higher quantum yield when decay would happen at this point. Indeed, of the eleven short living trajectories, nine readily reach the all-trans configuration (green squares in Fig. 4), and two retain the starting material (red triangles). This means that the chance for generating alltrans is significantly enhanced for hops at the first encounter ( $>80 \%$ ), but it drops to $c a$. $50 \%$ for hops at a later stage.

The effect of the hydrogen motions on the quantum yield can be understood by considering the $\pi$-overlap at the $\mathrm{C} 11=\mathrm{C} 12$ bond in the ground state (Fig. 5). The photoproduct will have maximum $\pi$-overlap along the $\mathrm{C}=\mathrm{C}$ axis. Fast hydrogen motion will cause pyramidalization of the formally $\mathrm{sp}^{2}$-hybridized $\mathrm{C} 11$ and $\mathrm{C} 12$ carbon centers, affecting the orientation of their $\pi$-orbital axis vectors (POAV). In POAV1 theory these vectors, which are related to the p-orbitals forming the $\mathrm{C} 11=\mathrm{C} 12 \pi$-bond, will form equal angles with the three $\sigma$-orbitals at the same atom. ${ }^{23}$ Thus, fast hydrogen motion will influence in which direction the $\pi$-overlap will be maximized right after the hop. Once the 

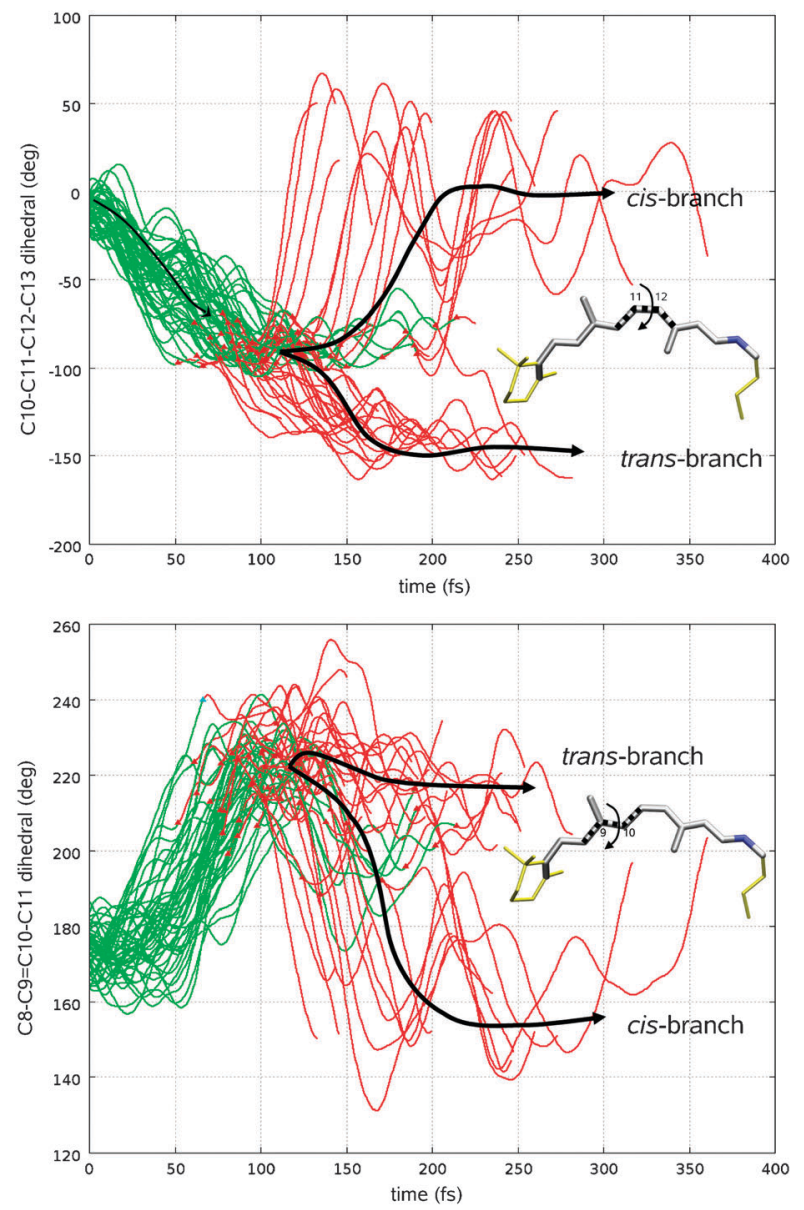

Fig. 2 Evolution of $\mathrm{C} 11=\mathrm{C} 12$ and $\mathrm{C} 9=\mathrm{C} 10$ torsion angles in individual trajectories. The green lines correspond to motion in the excited state, red triangles mark the event of surface hopping and the red lines denote ground state motion.
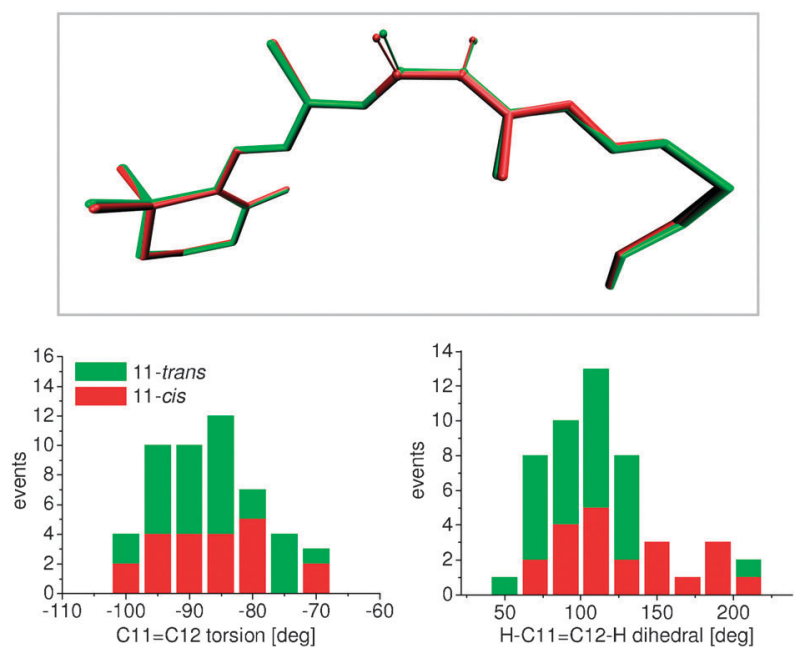

Fig. 3 Overlay of average hopping structures leading to the all-trans photoproduct (green) and back to 11-cis retinal (red), showing also hydrogens at $\mathrm{C} 11$ and $\mathrm{C} 12$, and distribution of $\mathrm{C} 10-\mathrm{C} 11=\mathrm{C} 12-\mathrm{C} 13$ and $\mathrm{H}-\mathrm{C} 11=\mathrm{C} 12-\mathrm{H}$ torsion angles. The portions of the bar graphs indicate the amount of cis or trans photoproducts.

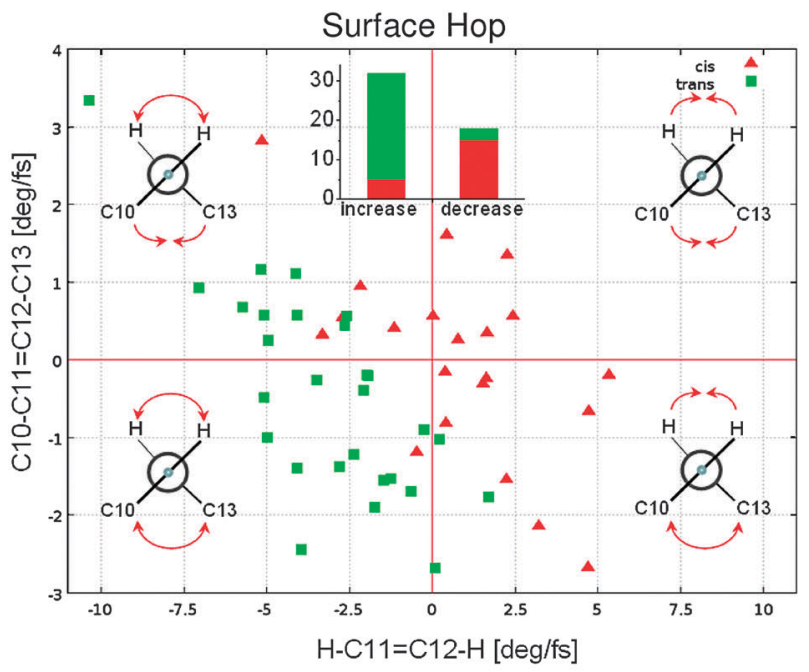

First $\mathrm{S}_{1} / \mathrm{S}_{0}$ approach

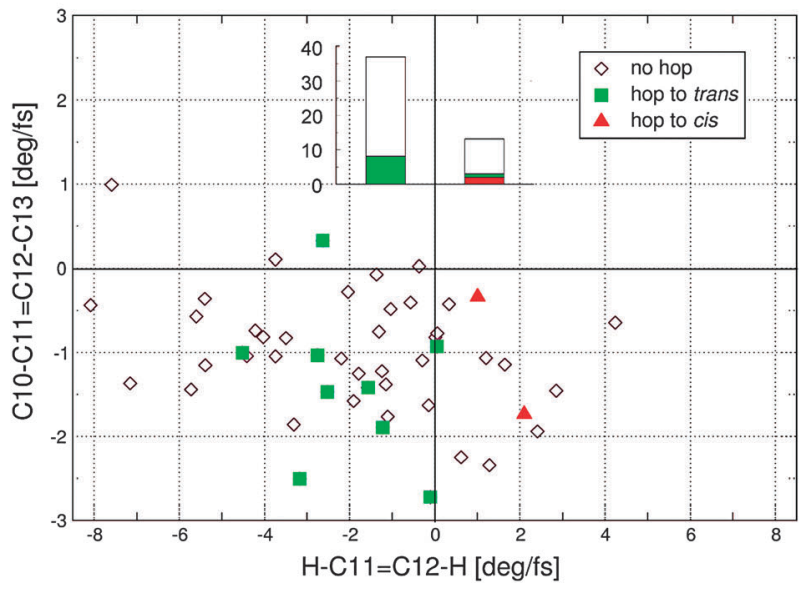

Fig. 4 Gradient of the $\mathrm{C} 10-\mathrm{C} 11=\mathrm{C} 12-\mathrm{C} 13$ and $\mathrm{H}-\mathrm{C} 11=\mathrm{C} 12-\mathrm{H}$ torsion angles at the hopping points (upper graph) and at the point of first close $\mathrm{S}_{1}-\mathrm{S}_{0}$ encounter. Green squares (red triangles) denote trajectories yielding the trans photoproduct (cis educt). Negative (positive) values correspond to increasing (decreasing) norm angles. Diamonds in the lower graph depict trajectories that do not hop at first close encounter of the two surfaces. The bar graphs count the number of events in the corresponding left and right halves of the diagrams.

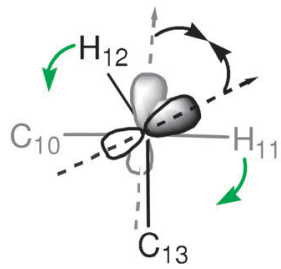

trans-photoproduct

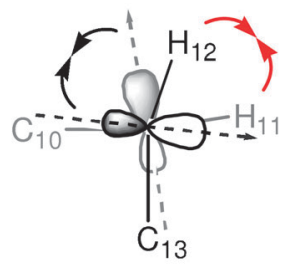

cis-photoproduct
Fig. 5 Relation between $\pi$-overlap and hydrogen motion at the $\mathrm{H}-\mathrm{C} 11=\mathrm{C} 12-\mathrm{H}$ moiety. The viewing direction is along the $\mathrm{C} 11=\mathrm{C} 12$ axis. $\mathrm{p}$-orbital axis vectors are tilted against the $\mathrm{C}-\mathrm{C}$ bonding axes and highlight the pyramidalization direction at $\mathrm{C} 11$ and $\mathrm{C} 12$ carbon centers.

double bond is restored, the carbon atoms will adjust to the bonding situation. In this picture hydrogen motion will lead to the all-trans-retinal isomer when, at the surface hop, the $\mathrm{C} 11=\mathrm{C} 12$ torsion is not significantly smaller than $90^{\circ}$ (we find 
no hops to $\mathrm{S}_{0}$ at values below $60^{\circ} \mathrm{C} 11=\mathrm{C} 12$ torsion) and $\mathrm{H}-\mathrm{C} 11=\mathrm{C} 12-\mathrm{H}$ torsion is increasing.

The $\mathrm{S}_{1} / \mathrm{S}_{0}$ non-adiabatic coupling vector at the point of surface hopping gives information on the geometrical distortions, which lift the energy degeneracy at the crossing point. Calculation of this property on selected hopping points indicates participation of HOOP motion in the coupling vector, i.e. this coordinate may also contribute in most effectively bringing the system toward the crossing and finally to the ground state (see SI).

In conclusion, we have shown that structural parameters alone are not sufficient to drive the retinal chromophore embedded in rhodopsin to a specific photoproduct. The direction of motion and, accordingly, the point of surface hopping are of significance. The light hydrogen atoms at the isomerising 11-cis double bond influence the outcome of the retinal photoreaction. Similar to trajectories of retinal models in vacuo, the $\mathrm{H}-\mathrm{C} 11=\mathrm{C} 12-\mathrm{H}$ coordinate in rhodopsin appears as a key mode in the photoproduct determining step. The decision which photoproduct is generated depends on the phase (i.e., the direction) of the hydrogen torsion. Pyramidalisation at the $\mathrm{C} 11$ and $\mathrm{C} 12$ carbon atoms due to fast hydrogen motion affects the alignment of p-orbitals, which form the $\pi$-bond of the photoproduct in the ground state. The photoproduct with the best overlap in the current direction of hydrogen motion will be the result of the photoisomerisation.

One may argue why most $(>60 \%)$ of the trajectories hop to $\mathrm{S}_{0}$ with hydrogen motion phases that are properly set to trigger the all-trans photoproduct. This happens because a significant fraction $(>20 \%$ ) of trajectories decays already at the very first encounter with the crossing seam, when the two $\mathrm{C} 11=\mathrm{C} 12$ torsional modes will mostly be still in phase, thus leading to a nearly fully efficient isomerization. For all other trajectories, that leave this region again and fail to decay fast, fast hydrogen oscillations lead to a dephasing between the two modes. Eventually, when they re-approach the crossing seam and decay to $\mathrm{S}_{0}$, a random photoisomerization occurs leading to a ca. $50 \%$ quantum yield.

This reasoning explains also the higher quantum yield and faster photoreaction reported in our earlier studies of pretwisted retinal models in vacuo. ${ }^{7,16}$ There, most of the PSB chromophores reach the conical intersection and hop to $\mathrm{S}_{0}$ at the first encounter of the two surfaces. This is different in $\mathrm{Rh}$, which has to account for many other effects necessary to turn retinal into a chromophore that absorbs at the correct wavelength. E.g., the placement of the counter-ion to shift the absorption maximum will, uncorrected by the protein, lead to an avoided crossing situation instead of a conical intersection, which itself will reduce the quantum yield. ${ }^{22}$ Still, the conversion in the protein pocket of $\mathrm{Rh}$ is ultrafast, unique and efficient. This is mainly achieved by shielding the electrostatic effects of the counterion that partially recovers the ideal in vacuo condition, ${ }^{24,25}$ and by the pre-twisted conformation of retinal toward the photoproduct side, which allows for fast and stereoselective motion along the torsion coordinate, the out-of plane motions of carbon skeleton and their corresponding hydrogens being in line after photoexcitation.

Finally, these findings open a new perspective in coherent control of ultrafast photoreactions involving polyenes: once the ensemble reaches the conical intersection region, a selective excitation of HOOP motion, e.g. by a laser pulse, can take major influence on the outcome of the isomerisation and may so effectively trigger or damp the photoreaction.

\section{Acknowledgements}

Part of this study was financially supported by the PRIN programme (2008JKBBK4). We thank Dr M. Barbatti for valuable discussion.

\section{Notes and references}

1 J. Bockaert, G. Roussignol, C. Becamel, S. Gavarini, L. Joubert, A. Dumuis, L. Fagni and P. Marin, Biochem. Soc. Trans., 2004, 32, $851-855$.

2 W. J. De Grip and K. J. Rothschild, in Molecular Mechanisms of Visual Transduction, ed. D. G Stavenga, W. J. DeGrip and E. N. Pugh, Elsevier, Amsterdam, The Netherlands, 2000, p. 1.

3 S. L. Logunov, L. Song and M. A. ElSayed, J. Phys. Chem., 1996, 100, 18586-18591.

4 F. Lumento, V. Zanirato, S. Fusi, E. Busi, L. Latterini, F. Elisei, A. Sinicropi, T. Andruniow, N. Ferre, R. Basosi and M. Olivucci, Angew. Chem., Int. Ed., 2007, 46, 414-420.

5 S. Sekharan, M. Sugihara, O. Weingart, T. Okada and V. Buss, J. Am. Chem. Soc., 2007, 129, 1052-1054.

6 A. M. Virshup, C. Punwong, T. V. Pogorelov, B. A. Lindquist, C. Ko and T. J. Martinez, J. Phys. Chem. B, 2009, 113, 3280-3291.

7 (a) O. Weingart, I. Schapiro and V. Buss, J. Mol. Model., 2006, 12, 713-721; (b) O. Weingart, J. Am. Chem. Soc., 2007, 129, 10618-10619.

8 (a) A. J. A. Aquino, M. Barbatti and H. Lischka, ChemPhysChem, 2006, 7, 2089-2096; (b) J. J. Szymczak, M. Barbatti and H. Lischka, J. Chem. Theory Comput., 2008, 4, 1189-1199.

9 M. Garavelli, T. Vreven, P. Celani, F. Bernardi, M. A. Robb and M. Olivucci, J. Am. Chem. Soc., 1998, 120, 1285-1288.

10 R. Gonzalez-Luque, M. Garavelli, F. Bernardi, M. Merchan, M. A. Robb and M. Olivucci, Proc. Natl. Acad. Sci. U. S. A, 2000, 97, 9379-9384.

11 L. M. Frutos, T. Andruniow, F. Santoro, N. Ferre and M. Olivucci, Proc. Natl. Acad. Sci. U. S. A., 2007, 104, 7764-7769.

12 S. Hayashi, E. Taikhorshid and K. Schulten, Biophys. J., 2009, 96, 403-416.

13 D. Polli, P. Altoe, O. Weingart, K. M. Spillane, C. Manzoni, D. Brida, G. Tomasello, G. Orlandi, P. Kukura, R. A. Mathies and M. Garavelli, Nature, 2010, 467, 440-443.

14 A. Warshel, Nature, 1976, 260, 679-683.

15 P. Kukura, D. W. McCamant, S. Yoon, D. B. Wandschneider and R. A. Mathies, Science, 2005, 310, 1006-1009.

16 O. Weingart, Chem. Phys., 2008, 349, 348-355.

17 P. Altoe, M. Stenta, A. Bottoni and M. Garavelli, Theor. Chem. Acc., 2007, 118, 219-240.

18 MOLPRO, version 2008.1, a package of ab initio programs, H.-J. Werner, P. J. Knowles, R. Lindh, F. R. Manby, M. Schütz, et al.

19 D. A. Case, T. E. Cheatham, T. Darden, H. Gohlke, R. Luo, K. M. Merz, A. Onufriev, C. Simmerling, B. Wang and R. J. Woods, J. Comput. Chem., 2005, 26, 1668-1688.

20 T. Okada, M. Sugihara, A. N. Bondar, M. Elstner, P. Entel and V. Buss, J. Mol. Biol., 2004, 342, 571-583.

21 E. Fabiano, G. Groenhof and W. Thiel, Chem. Phys., 2008, 351, 111-116.

22 G. Tomasello, G. Olaso-González, P. Altoè, M. Stenta, L. SerranoAndres, M. Merchan, G. Orlandi, A. Bottoni and M. Garavelli, J. Am. Chem. Soc., 2009, 131, 5172-5186.

23 R. C. Haddon, Pure Appl. Chem., 1986, 58, 137-142.

24 A. Cembran, F. Bernardi, M. Olivucci and M. Garavelli, J. Am. Chem. Soc., 2004, 126, 16018-16037.

25 J. Rajput, D. Rahbek, L. Andersen, A. Hirshfeld, M. Sheves, P. Altoè, G. Orlandi and M. Garavelli, Angew. Chem., Int. Ed., 2010, 49, 1790-1793. 